\title{
Prevención de riesgo psicosocial en adolescentes: el rolde los Centros de Día de Menores en España
}

\author{
Sergio Capella-Castillo, Ph.D. ${ }^{a}$ \\ Universitat de València, España \\ José Javier Navarro-Pérez, Ph.D. ${ }^{b}$ \\ Universitat de València, España
}

J.Javier.Navarro@uv.es

\section{Resumen (analítico)}

El artículo analiza la función de los Centros de Día de Menores (CDM) como recursos especializados en la intervención socioeducativa con adolescentes en riesgo. Se trata de una investigación cualitativa, en la que se han integrado diferentes técnicas con el fin de triangular evidencias objetivas. En el plano metodológico participó una muestra de 30 profesionales, implementando diferentes técnicas: entrevistas y grupos de discusión. Los datos obtenidos se codificaron y categorizaron. Los resultados destacan la especificidad de los CDM como recurso de proximidad en el proceso de socialización y de acompañamiento progresivo, individualizado y flexible de adolescentes en riesgo. Las conclusiones inciden en la especialización del recurso en la atención a adolescentes que atraviesan procesos vitales complejos y en la consolidación del recurso como alternativa socioeducativa a la educación formal, utilizando en el territorio como generador de oportunidades.

\section{Palabras clave}

Centros de Día de menores, adolescentes, riesgo social, prevención.

\section{Thesauro}

Tesauro de Ciencias Sociales de la Unesco.

\section{Para citar este artículo}

Capella-Castillo, S., \& Navarro-Pérez, J. J. (2021). Prevención de riesgo psicosocial en adolescentes: el rol de los Centros de Día de Menores en España. Revista Latinoamericana de Ciencias Sociales, Niñez y Juventud, 19(1), 1-22.

https://dx.doi.org/10.11600/rlcsnj.19.1.4293

\section{Historial}

Recibido: 13.01 .2020

Aceptado: 23.06 .2020

Publicado: 05.12.2020

Información artículo

El artículo es parte de la investigación denominada Factores de protección de los centros de día de menores: incidencia de la intervención socioeducativa con adolescentes en riesgo, presentada por el primer autor en acto público para optar el título de Doctor en Ciencias Sociales en la Universidad de Valencia (España) el 29/07/20 y que tuvo una calificación de Excelente Cum Laude. Trabajo dirigido por el Dr. José Javier Navarro-Pérez y la Doctora Mercedes Botija Yagüe, profesores e investigadores de la Universidad de Valencia. Área: Ciencias Sociales. Subárea: Psicología. 


\section{Prevention of psychosocial risk in adolescents: The role of Community Youth Centers in communities in Spain}

Abstract (analytical)

The article analyzes the role of Community Youth Centers (CDMs for their initials in Spanish) as a specialized resource for socio-educational interventions with at-risk adolescents. This is a qualitative investigation in which different techniques have been integrated in order to triangulate objective evidence. At the methodological level, 30 staff participated in different data collection processes (two discussion groups as well as semi-structured interviews). The data obtained was codified and categorized. The results highlight the specificity of the CDMs as an available resource for the process of socialization and the provision of gradual, progressive, individualized and flexible accompaniment to at-risk adolescents. The conclusions of the study recommend the specialization of the CDMs to support adolescents who are experiencing complex life processes and the consolidation of these centers as a socio-educational alternative that generates opportunities.

Keywords

Day Care Centers for Children, adolescents, social risks, prevention.

\section{Prevenção do risco psicossocial em adolescentes: O papel dos Centros de Dia Juvenis em Espanha}

\section{Resumo (analítico)}

O artigo analisa o papel desenvolvido pelos Centros de Dia de Menores (CDM) na intervenção socioeducativa com adolescentes em risco. Trata-se de uma investigação qualitativa, na qual diferentes técnicas foram implementadas para triangular evidências objetivas. Ao nível metodológico, 30 profissionais participaram em grupos de discussão e entrevistas semiestruturadas. Os resultados destacam a especificidade do CDM como um recurso de proximidade no acompanhamento gradual, progressivo, individualizado e flexível de adolescentes em risco. As conclusões incidem sobre a especialização deste recurso na educação de adolescentes nos seus processos de vida complexos e na sua consolidação como alternativa socioeducativa à educação formal.

Palavras-chave

Centros de dia para menores, adolescentes, risco social, prevenção.

Información autores

[a] Doctor en Ciencias Sociales. Profesor Asociado en la Facultad de Ciències Socials de la Universitat de València. H5: 0. iD 0000-0002-6261-7917. Correo electrónico: sergio.Capella@uv.es

[b] Doctor en Desarrollo Local y Territorio. Profesor Contratado Doctor del Departamento de Trabajo Social y Servicios Sociales. Investigador de la Red de Estudios sobre Juventud y Sociedad. Redes de Excelencia [CSO2017-90618-REDT]. H5: 8. (iD) 0000-0001-6363-7154. Correo electrónico: J.Javier.Navarro@uv.es 


\section{Introducción}

$\mathrm{E}_{\text {res }^{1} \text { de Valencia (España) como recurso de prevención ante situaciones de riesgo }}^{1 \text { presente artículo aborda el papel desarrollado por los Centros de Día de Meno- }}$ social. Por ello, nuestro interés por conocer las características intrínsecas del recurso y las dinámicas de intervención que ofrecen fortalezas y acciones preventivas frente a la denominada sociedad del riesgo (Beck, 1992), a partir de los y las profesionales que desarrollan diariamente su labor en estos recursos.

Si pretendemos adentrarnos en la realidad de las y los adolescentes vinculados de alguna manera con entornos de riesgo, se hace necesario conocer las expectativas, realidades de socialización, el papel de la formación obligatoria, las estructuras de ocio y tiempo libre, las pautas de recreo y sus canales de sociabilidad y consumo (Funes, 2009; Navarro-Pérez \& Puig, 2010).

Ante el actual escenario social, las políticas de prevención del estado español responden mediante el modelo gestión de riesgos, haciendo especial hincapié en la seguridad (Uceda \& Navarro-Pérez, 2013). Los modelos de gestión de riesgos se integran en la sociología del riesgo de Ullrich Beck (1992). Son paradigmas teóricos que analizan la realidad pero no intervienen sobre ella. El modelo de gestión de riesgos describe distintas dimensiones correlacionadas con las líneas estratégicas del humanismo de Nussbaum (2012) o los lineamientos sobre el equilibrio del bienestar de Perdomo y Valera (2015).

La importancia de establecer políticas de prevención ante situaciones consideradas de riesgo en adolescentes procede de una estrategia que refleja «intervenciones preventivas [que] tienen una eficacia y rentabilidad superior a medio y largo plazo mayor que aquellas dirigidas a la rehabilitación cuando el problema ya ha hecho su aparición» (Arruabarrena, 2001, p. 143). Por tanto, las estrategias preventivas inciden en el desarrollo de actividades con mayores opciones de éxito (Gresham, 2016). La labor de prevención y

\footnotetext{
${ }^{1}$ En adelante CDM.
} 
protección no se entiende solamente ante la aparición de situaciones de riesgo. Por ello, se hace necesaria la instauración de bases sólidas que permitan el establecimiento de entornos de seguridad, facilitando un desarrollo integral de las y los adolescentes (Jiménez, 2016). Por ello, y de acuerdo con Zambrano et al. (2015), el ámbito comunitario donde se establece el CDM, emerge como engranaje convivencial seguro, con capacidad para equilibrar los desequilibrios territoriales donde confluyen los diferentes agentes sociales.

\section{Proceso de socialización y prevención ante situaciones de riesgo en la adolescencia}

Desde la década de los años ochenta del pasado siglo se ha ido produciendo un importante cambio: los niños y niñas de entre doce y diecisiete años tenían la obligación de ser adolescentes, dándoles a entender que tenían unos años por delante para eludir responsabilidades propias de la edad adulta (Funes, 2009).

Para referir a las situaciones de riesgo social, se alude a todas aquellas coyunturas y condiciones personales, familiares o sociales que incrementan la atracción de los adolescentes para asumir dificultades mediatas o directas (Castro \& Rodríguez, 2016; González, 2006), ejerciendo influencia negativa en su desarrollo personal.

El proceso de socialización de los adolescentes supone un transcurso de interacciones entre la sociedad total y los agentes de socialización (Berger \& Luckman, 1995) donde, a través de la asimilación de conocimientos y costumbres, se adquieren valores y sentimientos de forma no consciente. Tales trayectorias, no exentas de riesgos, originan itinerarios en los que se aprecian nuevas experiencias, sensaciones y estímulos (Steinberg et al., 2017), donde la ruptura con los comportamientos prosociales es consecuencia de la socialización con entornos y prácticas consideradas de riesgo (Navarro-Pérez \& Pastor, 2018).

\section{Los Centros de Día de Menores de la Comunitat Valenciana: un recurso preventivo de atención a la adolescencia}

La atención social dirigida a la infancia ha sido una ocupación persistente en el tiempo para los poderes públicos, como ponen de manifiesto las referencias halladas desde los tiempos de la España hispanorromana (Martínez, 2002). La primera década del siglo XX en España supuso una autentica mejora en materia de protección infantil, llegando así a la década de los años setenta en la que se localiza el primer antecedente de los 
CDM, denominado Centre Obert Joan Salvador Gavina, situado en la ciudad de Barcelona, y perteneciente a una iniciativa privada (Biosca \& Casas, 1986).

Sánchez (2011) afirma que «utilizar el término Centros de Día puede tender a confusión si no se especifica a qué colectivo va dirigido» (p. 120). En el caso de la Comunidad Valenciana, la Ley 26 de derechos y garantías de la infancia y la adolescencia (Generalitat Valenciana, 2018) regula el reconocimiento en relación de las atribuciones hacia la infancia y la adolescencia, señalando el principio de corresponsabilidad de actuación entre la sociedad y las administraciones públicas, así como el marco normativo para definir las políticas públicas, distribución de competencias y medidas de coordinación.

Los centros de atención diurna - en este caso CDM - son determinados como centros de protección de niños, niñas y adolescentes, «cuyo objetivo principal es ofrecer, a niños y adolescentes en situación carencial, la posibilidad de aprovechamiento y aprendizaje, mediante medidas de distinta índole (de promoción personal, ocupacionales formativas, socioculturales, prelaborales, rehabilitadoras y lúdicas). Siendo un recurso para la prevención de situaciones de riesgo social» (Ferrero, 2012, p. 37).

En anteriores actualizaciones legislativas, la función preventiva del CDM ya emergió como eje de la intervención socioeducativa (Montoliu, 2013), definiendo esta tipología de recurso como «estructuras preventivas, recomendables para menores que necesitan un apoyo a la socialización en su propio medio, bien porque manifiestan comportamientos de inadaptación social, o porque culminan un proceso de acogimiento residencial y necesitan una orientación multifacética» (p. 6). De este modo, los niños/as y adolescentes usuarios de los CDM son aquellos que viven situaciones de riesgo o exclusión social (Montoliu, 2013).

Frente a lo anterior, el desarrollo metodológico se orientó a efectos de alcanzar los siguientes objetivos:

1. Determinar las características particulares de los CDM que colaboran en la implementación de propuestas de intervención socioeducativa.

2. Identificar las fortalezas y oportunidades que posibilitan acciones preventivas desde los CDM.

En la línea de lo expuesto, surgen preguntas de investigación que facilitaran la etapa empírica: ¿cómo se construyen estos servicios educativos?, ¿cómo se insertan en la educación? y ¿qué tipo de acciones preventivas desarrollan? 


\section{Método}

Se realizaron dos grupos de discusión y 17 entrevistas semiestructuradas. El uso de distintas técnicas para la consecución de los objetivos de investigación permitió la triangulación metodológica intramétodo (Denzin, 1970). A partir del grupo de discusión I se empleó un debate guiado por un moderador (Navarro-Pérez, 2014), en el que se generaron detalles en torno a razonamientos y experiencias complejas. El grupo de discusión I (tabla 1) estuvo formado por informantes-expertos y pretendió establecer las áreas e indicadores a investigar en el procedimiento empírico, los criterios de inclusión muestral y los puntos de interés a desarrollar en las entrevistas semiestructuradas. Así, se recopiló la información a partir de la opinión de profesionales en la atención directa de la propia realidad investigada (Kvale, 2011).

\section{Tabla 1}

\section{Participantes del grupo de discusión I}

\begin{tabular}{rlclc}
\hline $\mathbf{N}^{\circ}$ & Participante & Código & \multicolumn{1}{c}{ Institución de origen } & Duración \\
\hline 1 & Educador social & GDI1 & CDM zona atención preferente & \\
2 & Directora & GDI2 & CDM urbano \\
3 & Trabajador social & GDI3 & CDM rural & \\
4 & Psicóloga & GDI4 & Instituto secundaria (educación formal) & 1:37' \\
5 & Educadora social & GDI5 & Centro Justicia Juvenil \\
6 & Educadora social & GDI6 & Ayuntamiento población rural \\
7 & Profesor universitario & GDI7 & Universidad de Valencia \\
\hline
\end{tabular}

Derivado de la instrumentalización del grupo de discusión I, los informantes-expertos enumeraron los siguientes criterios de inclusión:

1. El compromiso voluntario, libre y autónomo de los profesionales en la investigación.

2. Los profesionales participantes debían desarrollar su labor diaria en el CDM y en contacto directo con los adolescentes (educadores/as sociales, trabajadores/as sociales, psicólogos/as, directores/as y maestros/as taller). 
3. Los profesionales deberían acreditar una experiencia superior a cinco años en el desarrollo de actividades socioeducativas en un CDM o en asociaciones educativas de similares características.

4. El establecimiento de la paridad de género en la elección de los informantes clave.

De esta manera, el grupo de discusión I ofreció resultados en la forma de orientación para la práctica empírica posterior.

A continuación, y estableciendo la complementariedad de técnicas de investigación, se utilizó la entrevista semiestructurada para dotar de una amplia heterogeneidad en el dibujo discursivo y garantizar «el multienfoque» (Navarro-Pérez \& Pastor, 2018, p. 125). Se reunieron — según recoge la tabla 2 - diferentes perfiles profesionales de informantes (Coulthard, 1998).

Tabla 2

Codificación de expertos/as entrevistados y área de atención

\begin{tabular}{llllc}
\hline $\mathbf{N}^{\circ}$ & \multicolumn{1}{c}{ Área } & \multicolumn{1}{c}{ Expertos/as } & \multicolumn{1}{c}{ Institución de origen } & Código \\
\hline 1 & Servicios sociales y bienestar & Educador social & Asociación educativa & E1 \\
2 & Servicios sociales y bienestar & Director & CDM público 1 (urbano) & E2 \\
3 & Servicios sociales y bienestar & Educadora social & CDM concertado 2 (rural) & E3 \\
4 & Servicios sociales y bienestar & Maestro taller & CDM público 1 (urbano) & E4 \\
5 & Servicios sociales y bienestar & Educador social & CDM público 1 (urbano) & E5 \\
6 & Servicios sociales y bienestar & Educador social & CDM concertado 3 (urbano) & E6 \\
7 & Servicios sociales y bienestar & Educadora social & CDM concertado 4 (urbano) & E7 \\
8 & Educación y empleo & Educadora social & CDM concertado 4 (urbano) & E8 \\
9 & Servicios sociales y bienestar & Director & CDM concertado 5 (urbano) & E9 \\
10 & Servicios sociales y bienestar & Trabajadora social & CDM concertado 5 (urbano) & E10 \\
11 & Servicios sociales y bienestar & Trabajadora social & CDM concertado 6 (urbano) & E11 \\
12 & Servicios sociales y bienestar & Directora & CDM concertado 2 (rural) & E12 \\
13 & Servicios sociales y bienestar & Psicóloga & CDM concertado 4 (urbano) & E13 \\
14 & Educación y empleo & Psicólogo & CDM concertado 6 (urbano) & E14 \\
15 & Servicios sociales y bienestar & Directora & CDM concertado 6 (urbano) & E15 \\
16 & Servicios sociales y bienestar & Maestro taller & CDM público 7 (rural) & E16 \\
17 & Servicios sociales y bienestar & Trabajadora social & CDM público 7 (rural) & E17 \\
\hline
\end{tabular}


Los hallazgos de las entrevistas se utilizaron para profundizar en el conocimiento del recurso (Wengraf, 2012). Se abordaron indicadores relativos al tipo de funcionamiento de los CDM, el papel del recurso y el perfil de adolescentes atendidos, o la gestión y planificación de las intervenciones socioeducativas llevadas a cabo con los adolescentes. Posteriormente, estos hallazgos se codificaron mediante segmentos de texto, dando como resultado las principales categorías y subcategorías (tabla 3).

Tabla 3

Categorías y subcategorías derivadas de las Entrevistas semiestructuradas

\begin{tabular}{lll}
\hline $\mathbf{N} .^{\circ}$ & \multicolumn{1}{c}{ Categoría } \\
\hline \multirow{2}{*}{ Especificidad: recurso de intervención } & \multicolumn{1}{c}{ 1.1. Acompañamiento vital } \\
& 1.2. Integración en el sistema de bienestar \\
& 1.3. Dimensión territorial \\
& 2.1. Hábitos y vínculos \\
& 2.2. Flexibilidad procesos \\
& 2.3. Estrategias: evaluación continua, motivación, \\
& responsabilidad, etc. \\
& 2.4. Conflicto como oportunidad: procesos \\
& 2.5. Aprendizaje experiencial-contextual \\
& 2.6. Robustecimiento de lazos: grupo educativo
\end{tabular}

Atendiendo las recomendaciones de Parker y Tritter (2007), las categorizaciones resultantes se sistematizaron y se reaplicaron al grupo de discusión II (tabla 4), con el propósito de validarlas o refutarlas y, de tal manera, alcanzar la objetividad de los hallazgos.

Tabla 4

Participantes e institución del grupo de discusión II

\begin{tabular}{clclc}
\hline N. & \multicolumn{1}{c}{ Participante } & Código & \multicolumn{1}{c}{ Institución de origen } & Duración \\
\hline 8 & Trabajadora social & GDII1 & CDM zona centro \\
9 & Director & GDII2 & Centro de Acogida de Menores provinciano & \\
10 & Psicólogo & GDII3 & CDM área metropolitana & \\
11 & $\begin{array}{l}\text { Presidenta Colegio Oficial } \\
\text { Educadores Sociales }\end{array}$ & GDII4 & Asociación Profesional de Educadores Sociales & 1:56' \\
12 & Educadora social & GDII5 & CDM zona periférica \\
13 & Educador social & GDIl6 & CDM zona atención preferente \\
\hline
\end{tabular}


En esta línea, Lederman (1990) destaca la importancia de esta técnica para evaluar la eficacia analítica derivada de la segregación de contenidos. Posteriormente, se siguieron las recomendaciones de Morse (1995) relativas a la saturación de la información al no hallar nuevos resultados en el proceso empírico; este factor permitió construir una teoría comprehensiva y convincente sobre el objeto de estudio. Apoyados en la dimensión estructural de Buttram (1990), este proceso secuencial ayudó en la sistematización objetiva de los resultados.

El tratamiento textual, procesual y comprensivo derivado de la utilización de las diferentes técnicas se llevó a cabo mediante el software MAXqda 12, sistematizando dos agrupaciones de análisis: texto y concepto. Para el presente artículo se recuperaron 107 segmentos de texto, que finalmente pudieron ser reproducidos poco más de $1 / 3$, integrándose las recomendaciones de Legreco y Tracey (2009) con objeto de promover heterogeneidad discursiva, que permitiese dotar de una dimensión explicativa a las categorías de análisis. ${ }^{2}$

Desde los planteamientos de responsabilidad ética de Abebe y Bessell (2014), se obtuvo el compromiso y el consentimiento informado de todos los profesionales, a efectos que decidiesen autónoma, libre y voluntariamente su participación en la investigación. Sus opiniones fueron tratadas para fines exclusivos de investigación.

\section{Resultados}

Para este artículo, se plantearon dos objetivos: 1) determinar las características particulares de los CDM que colaboran en la implementación de propuestas de intervención socioeducativa; 2) identificar las fortalezas y oportunidades que posibilitan acciones preventivas desde los CDM. En relación al primero de ellos, se obtuvieron los siguientes resultados:

\section{El acompañamiento vital en el desarrollo de la adolescencia}

Los CDM son recursos del Sistema de Bienestar Social. La estructura y profesionalización mostradas y el rigor de los sistemas de calidad a los que son sometidos, les dotan

\footnotetext{
${ }^{2}$ Cabe destacar que surgen más categorías de análisis derivadas de las técnicas de investigación, pero debido a
} las restricciones de extensión se han considerado aquellas que respondían a los objetivos planteados. 
de un adecuado sistema de funcionamiento, el cual les caracteriza como los recursos principales dirigidos al apoyo y atención de la infancia y adolescencia.

Los Centros de Día son recursos de mucha calidad. Se han regulado y protocolizado las actuaciones. $\left(\mathrm{E}_{7}\right)$

Llevamos unos años en que nos hemos profesionalizado, donde hemos hecho una intervención mucho más profesional y organizada; hemos hecho un esfuerzo importante de que sea un recurso profesional que tenga un valor. (E12)

El hecho de establecer una serie de procesos y de hacernos conscientes de como hacíamos las cosas. Los sistemas de calidad, no es [sic] solamente una carga, sino que supone un gran beneficio para nosotros, para equiparar los criterios de intervención. (GDII6)

Un elemento que destaca como particularidad de la intervención de los CDM se halló en el ambiente generado dentro del recurso. El espacio de tranquilidad con el que se interviene con los y las adolescentes supone una característica fundamental que facilita su proceso de inclusión.

También se encuentran en un ambiente en el que son aceptados; eso es importantísimo, ya que su autoestima ahí se refuerza. No son los malos de la clase, no son los chungos... sino que pueden tener otro papel. (E13)

Se cuida hasta el más mínimo detalle. Desde las cortinas a quien es la persona que les abre la puerta (...) un ambiente curativo. (GDII6)

\section{El CDM como recurso del sistema de servicios} sociales, ámbito comunitario y afectivo

Los CDM se encuentran integrados como recurso de atención desde los sistemas de bienestar. La intervención desarrollada es capital en la atención a la dificultad desde el propio contexto de los y las adolescentes. Por ello, mantienen vínculos directos con los servicios de atención comunitaria y el acceso a los mismos se realiza a partir de protocolos de derivación institucional, en coordinación con otros sistemas de bienestar: servicios sociales, educación, empleo, justicia juvenil, etc.

Formamos parte de una red especializada de recursos de atención directa a infancia y adolescencia. (E14)

Ya no le extraña a nadie que desde los CDM nos coordinemos con los centros de secundaria o los itinerarios de empleo con apoyo. Somos una pata más del bienestar. (E6) 
Las y los expertos también reparan en la proximidad hacia el propio espacio de los y las adolescentes. La intervención y las dinámicas socioeducativas ofrecen un trato cercano y de confianza, donde se afianzan unos límites de acción positivos.

La cercanía la necesitas para ganarte la confianza del chaval, pero siempre dejando claro los límites. Es decir, «yo no soy tu colega, tu amigo; yo estoy aquí para lo que estoy, es decir, para ayudarte en la medida de lo posible. Pero, por otro lado, yo voy a informar lo que haces bien y mal. Mi papel es ayudarte a que hagas todo lo bien posible, para que los informes sean positivos». (E4)

Un cocktail de normas y cariño en un ambiente constructivo. (E3)

Estamos muy próximos al día a día. Vemos diariamente a los chavales, con lo cual tenemos más cercanía. (E11)

Así mismo, los CDM son recursos integrados en el propio barrio, y esto supone una clave fundamental para su labor. La posibilidad de realizar la intervención en el propio contexto del adolescente, poseer un conocimiento acerca del entorno y estar conectados a su lugar de desarrollo, conlleva una amplitud de trabajo que resulta diferencial para una intervención efectiva.

Es mucho más provechoso donde podemos tener una relación, no solamente con el menor, sino con su ámbito de iguales. (E1)

Es una tela de araña que generamos con la escuela, grupo de iguales (...). El estar integrados en el barrio facilita el acceso a otros recursos que propicia que el chaval dé pequeños pasos, pero siempre hacia delante. (GDII 4$)$

El conocimiento previo que los y las adolescentes poseen de los CDM supone un factor fundamental para el inicio de la andadura. La aceptación sobre del recurso que ofrece el barrio produce un sentimiento de confianza hacia la intervención como apoyo al crecimiento prosocial de los y las adolescentes. Los CDM asumen una serie de compromisos en sus respectivos barrios que los convierten en referentes de la zona en la que desarrollan su actividad.

¿Qué sucede, qué hacen los Centros de Día? Pues es una red social; es un lugar que aglutina y es una referencia en el barrio. Ayuda a toda la población, no solo a los menores que vienen. (Eg) 
El hecho que los CDM estén en su territorio les ayuda a generar nuevos vínculos y asegurar su zona de confort. (E10)

Otra cuestión a destacar es la vinculación con la relación educativa que se establece en los CDM. Las dinámicas de acompañamiento parten desde relaciones educativas asociadas al afecto, al amor, a la comprensión y al acompañamiento. De esta forma, dicha configuración de las relaciones educativas, exentas de la rigidez que se ofrece en otros ámbitos, implica un bienestar y la posibilidad de trabajar desde procesos educativos individualizados.

Cuando vienen al Centro es una oportunidad que tienen; es alguien que me quiere como soy, no me rechazan, no me expulsan por como soy..., sino que analiza cuál es mi necesidad, por qué me muevo como me muevo, por qué tengo los problemas que tengo en mi casa, y a partir de ahí vamos a trabajar. (Eg)

En el colegio las dinámicas se generan en función del grupo-aula, y las tutorías no permiten esta intervención personal. (GDII6)

El segundo de los objetivos profundizó en la identificación de fortalezas y oportunidades que posibilitan acciones preventivas desde los CDM. Atendiendo a ello presentamos las siguientes aportaciones:

\section{Hábitos para construir procesos vitales seguros}

Los CDM ofrecen espacios de socialización seguros, donde los y las adolescentes establecen ambientes de confianza y pueden expresar sentimientos y actitudes que les resulta más difícil desde otros contextos. Los y las adolescentes informantes clave también refieren a la ascendencia del recurso para la eclosión de sus capacidades. De esta forma, los y las adolescentes entienden el CDM como un espacio de autonomía en las formas de relación que favorece vínculos de seguridad para afrontar sus situaciones.

Es un espacio donde ellos se sienten seguros a nivel afectivo, y que pueden contar con nosotros. (E6)

Trabajamos el itinerario en el que se van definiendo poco a poco. Pero llega un punto en el que han hecho techo y nuestra idiosincrasia hace que se promulgue la autonomía y no se acomoden para que no retrocedan. (GDII1) 
La capacidad de negociación que muestra el recurso supone una fortaleza según señalan las y los expertos. Desde los CDM se pretende aportar respuestas y soluciones ante las carencias detectadas, que otros recursos integrados en el territorio no pueden llevar a cabo.

Tiene que saber dar respuestas a todas aquellas carencias que las estructuras normalizadas no pueden llegar, o no saben dar y no están preparadas para ello. Entonces, el Centro de Día tiene que tener esa opción de poder crear. (E1)

Es fantástico que interpreten el CDM como un espacio de crecimiento para revertir sus miedos, conflictos y fracasos en confianza y estímulos de progreso. (E10)

Los CDM ofrecen dinamismo y flexibilidad, lo que supone un espacio de escucha, crítica y reflexión en el acompañamiento diario con los y las adolescentes.

Cuando tú le aportas alternativas, incluso le puedes hablar de esos temas con cierta naturalidad y cierta familiaridad, en el fondo les gusta, porque en su entorno nadie les hace reflexionar. $\left(\mathrm{E}_{4}\right)$

Provocarles la crítica. Que no se crean todo lo que ven en Instagram y en el YouTube. (E1o) Nosotros tenemos que ser un trampolín (...). Estamos aquí para que te reubiques, te motives y tires para el lado que te guste y te motive. (GDI2)

\section{Conflicto como oportunidad}

La conflictividad sufrida en la adolescencia viene derivada por situaciones de desarraigo, de choque cultural, ausencia de estímulos o fracturas afectivas. Los CDM están consolidados como recurso para atajar las situaciones de conflicto social o escolar. De esta forma, generan oportunidades adaptadas a la situación y posibilidad de cada adolescente, para paulatinamente incrementar sus competencias.

El menor comparte experiencias intensas de convivencia y satisfacción. Eso es una oportunidad (...), otras formas de querer, otras formas de hablar, otras formas de resolver conflictos (...). Y esto son distintas situaciones de crecimiento para el chaval. (E12) Conflictos para plantear alternativas. Conflicto para aprender. (GDII3)

El actual sistema educativo se plantea a través de itinerarios adaptativos, donde el niño se aclimata al sistema, y no a la inversa. En este sentido, el CDM ofrece un espacio educativo inclusivo, en el cual se trabaja desde diferentes líneas de actuación a nivel 
individual y grupal, llevando a cabo actividades complementarias al sistema educativo. Las y los expertos exponen que esta labor no implica un nivel inferior de exigencia, sino que, con un ritmo adaptado, supone otra oportunidad de obtener el proceso de aprendizaje requerido, en consonancia con los objetivos curriculares, laborales y competenciales que tienen asignados.

Contribuyes mucho a que ese niño empiece un itinerario mucho más positivo. Puedes darle nuevas herramientas y oportunidades. $\left(\mathrm{E}_{4}\right)$

El Sistema de Educación y el de Bienestar Social tienen que trabajar en común y abrirse puertas uno al otro. (GDI4)

Nuestro trabajo de complementación y de colaboración, más que de alternativo al sistema educativo. (GDII3)

\section{Trabajo motivacional, progresivo y constante para el desarrollo de la responsabilidad}

En este recurso el trabajo desde la motivación desempeña un rol importante. De esta manera, el CDM muestra una capacidad para transformar la obligación en compromiso y responsabilidad para la asistencia de los y las adolescentes, convirtiéndose en una de las principales fortalezas del recurso.

El trabajo es hacer que quieran venir. Para mí el trabajo está ahí, porque obligarlos, ya les obliga todo el mundo a hacer muchas cosas. (E6)

Les cambia mucho por la propia metodología del Centro de Día. (E14)

Cuando un adolescente llega al CDM inicia un proceso de aceptación del recurso. La acogida inicial es fundamental para superar posibles barreras hacia la intervención y, posteriormente, cada pequeño paso supone un avance, siendo otra de las singularidades de los CDM.

Todos los que trabajamos con nanos nos tenemos que pasar cuatro o cinco sesiones en las que tú estas diciendo a los padres: «no os preocupéis, estoy ganándomelo»; porque claro, si no tienen una confianza contigo, no te va a contar nada. (E10) Más vale pasos cortos e intensos que grandes e inestables. (GDI1) 
El CDM emerge como un espacio de prevención donde el adolescente adquiere pautas de desarrollo positivas, dirigidas a evitar factores de riesgo, que resultan determinantes en la orientación de sus vidas, en la búsqueda de nuevos objetivos y en la asunción de responsabilidades. El cumplimiento de rutinas y de un horario estipulado beneficia la consecución de este propósito.

La mayoría de chavales y chavalas que entran a un Centro de Día encuentran a alguien que, a veces, hasta se asimila a ellos y, por tanto, se hallan más cómodos en esa realidad. (E3) Los chavales siguen durante años conectados al recurso porque la responsabilidad no es como una asignatura que se consiguen los objetivos curriculares y ya está. La responsabilidad se tiene que seguir cultivando porque, si no, se marchita. (GDII 4 )

\section{El grupo educativo: recurso colateral que fortalece la intervención}

El CDM ofrece un punto de encuentro y consenso: el grupo educativo. Éste constituye una dinámica de trabajo que radica en la consistencia grupal y en la pertinencia a través de vínculos y otras referencias prosociales.

Trabajamos mucho el grupo educativo, el grupo de referencia. Hay cosas que tienen más sentido desde el trabajo grupal. (E11)

Una de las claves es la retroalimentación que cada chaval recibe de sus compañeros (...); lo notan y favorece su crecimiento. (GDII5)

Las y los expertos indican que el grupo educativo enriquece la dinámica del recurso. El grupo aporta comunicación, consenso y un punto de coincidencia entre los y las adolescentes, a partir del cual se establecen los ejes a seguir durante las rutinas diarias. El grupo actúa en muchos casos de modulador de las normas y límites, favoreciendo la diversidad de espacios constructivos e instrumentalizando el grupo educativo como marco de referencia.

Participar en dinámicas de grupo, el tema de hábitos, de normas en positivo, de funcionamiento estructurado constituyen factores de protección al chaval. (E2)

Ninguna decisión individual está por encima de acuerdos grupales. (E7)

También está la autorregulación del grupo. (E8) 
Por ello, el grupo educativo fomenta los sentimientos de trabajo en equipo, solidaridad, compromiso, responsabilidad, entendimiento y compenetración que, de forma transversal, son oportunidades para el desarrollo positivo.

\section{Discusión}

Para este trabajo se han planteado dos objetivos: el primero de ellos consistió en determinar las características propias de recursos de atención especializada como son los $\mathrm{CDM}$, los cuales colaboran en la implementación de propuestas de intervención socioeducativa. El segundo trató de identificar las fortalezas y oportunidades que permiten a los CDM impulsar acciones preventivas. Debido a ello, nos interesó analizar el tipo de recurso en primer lugar y, seguidamente, identificar las fortalezas que permiten intervenir desde una coyuntura preventiva, capaz de preservar riesgos y orientar en la convivencia prosocial de los y las adolescentes. En relación con el primer objetivo, los CDM han experimentado un grado de profesionalización en su labor, situándose dentro del Sistema de Servicios Sociales. En relación a ello, nuestros resultados coinciden con las aportaciones de Ferrero (2012), que incide una organización y estructura sólida del recurso, consolidándose como pieza clave para el trabajo social, personal y escolar. Es en este sentido en que se justifica la integración en el territorio de los CDM, como recurso para promover la prevención ante factores de riesgo (Ciocanel et al., 2017; Santibañez \& Laespada, 2016).

En la actualidad poco se ha investigado acerca de la intervención socioeducativa del CDM y su función preventiva. Por ello, investigaciones como las que se presentan en este artículo ofrecen nuevas alternativas para hacer frente a situaciones de riesgo. En este sentido, se muestran fortalezas que ofrece la labor realizada por este recurso, que no solo previene de situaciones de riesgo, si no que genera hábitos y responsabilidades en los usuarios para fomentar el desarrollo de la propia autonomía en su contexto.

\section{Espacio de proximidad, referente, afectivo y de acompañamiento}

En los resultados, se observa la función del CDM como recurso principal de las políticas de prevención ante situaciones de riesgo para la infancia y la adolescencia. El papel de acompañamiento llevado a cabo se pone de manifiesto en el contacto diario con el 
equipo educativo (Jiménez, 2016). En esta línea, la labor ofrecida desde el CDM se centra en la persona, desde donde se atiende, entiende y sufre con el adolescente, antes de encontrar respuestas inmediatas y poco consensuadas. Esta fortaleza concuerda con los resultados obtenidos por Tur et al. (2016), que concluyen la necesidad de establecer programas cercanos y altruistas que se caractericen por un acercamiento afectivo.

Los hallazgos evidencian que el acompañamiento ejercido desde el recurso promueve relaciones desde el afecto y el amor. Esto coincide con los resultados de investigación de Navarro-Pérez y Puig (2010), donde el CDM se hace conocedor de las realidades y canales socializadoras del adolescente, que confluyen para establecer pautas de ocio prosociales. Además, la integración de los CDM en el territorio propio de los y las adolescentes facilita un trabajo directo.

La intervención socioeducativa de los CDM reconforta al adolescente en su crecimiento personal, ya que no se siente juzgado, sino que se eleva como protagonista ante una estructura de apoyo en su entorno comunitario. En este sentido, coincidimos con Vallejo et al. (2017) en relacionar la participación comunitaria con una esperanza de cambio. Por el contrario, una actitud rígida se asocia a actitudes fatalistas. El CDM se caracteriza de forma esencial por tener en consideración las potencialidades del contexto de los adolescentes ya que, de acuerdo con Orrego et al. (2015), las conductas de riesgo en general asoman mediante la imitación de pautas del entorno cercano.

Relacionado con el segundo objetivo, y como cuestión de fondo, en este artículo se cuestiona si son los CDM recursos efectivos para la articulación de acciones preventivas con adolescentes en riesgo. En esta línea, el desarrollo individual, grupal y comunitario es la meta que persiguen los CDM, y coincidimos con Ruiz y Palma (2019) en integrar la perspectiva laboral en clave de promoción para el desarrollo de estrategias de autonomía durante la intervención.

\section{La prevención como oportunidad en la intervención socioeducativa desde los CDM}

Se ha observado en los resultados que los CDM actúan como «colchón» para amortiguar y reducir los mecanismos de riesgo. Por ello, coincidimos con la función preventiva descrita en el estudio de CDM de Sánchez (2011), que se realiza bajo el abastecimiento de patrones formales de integración social y escolar. En este sentido, los CDM preparan al adolescente hacia nuevos retos, donde el desarrollo de sus competencias pueda integrarlos. 
El dinamismo y flexibilidad mostrado por los CDM permite a los adolescentes adquirir estrategias y empoderarse para afrontar un proyecto vital con mayores garantías de éxito (Ferrero, 2012; Uceda, 2011). Los resultados obtenidos mostraron esta percepción en analogía a la investigación de Oberle et al. (2011) mediante el desarrollo de competencias a partir de estrategias basadas en la resiliencia.

Las acciones preventivas de los CDM nos conducen al trabajo en el entorno, como oportunidad de aprendizaje y conocimiento en los y las adolescentes. Nuestros hallazgos son análogos a investigaciones que diagnostican la función de aprendizaje (Montoliu, 2013) como eje sobre el que se sostiene la intervención del recurso. En esta línea, y de acuerdo con Melendro et al. (2017), los resultados nos muestran la importancia de un trabajo motivacional continuado, que produce mayor participación de los adolescentes en el proceso.

Por último, las estrategias educativas grupales fomentan mayor implicación de los y las adolescentes, impulsando la creatividad y el sentido de la responsabilidad. Nuestros resultados coinciden con Figueiredo (2016) quien destaca la intervención socioeducativa grupal como factor de apoyo en el desarrollo personal, entre los que incluye la capacidad de resiliencia de los y las jóvenes. Así mismo, las conclusiones nos muestran como, desde el trabajo grupal del CDM, se establecen relaciones sociales bajo el umbral normativo, las cuales posibilitan hábitos prosociales y aprendizajes complementarios. El grupo educativo genera participación, discusión y aprendizajes que favorecen la autonomía y el desarrollo personal.

\section{Limitaciones y aplicaciones prácticas}

Las limitaciones de este artículo principalmente se centran en la discusión, ya que la literatura científica apenas ha investigado los dispositivos de prevención de situaciones de riesgo social instalados en la comunidad. Por tanto, las reducidas experiencias han limitado una discusión comparada de mayor calado y crítica. Por otro lado, la investigación se contextualiza en una zona geográfica concreta con unos marcos jurídicos delimitados que de alguna manera impiden comparar exhaustivamente este tipo de recurso con otros de similares características en otras regiones de España, en particular en el País Vasco y Navarra donde se desarrollan políticas de prevención pública con larga tradición en materia de niñez y juventud.

El presente trabajo deja en herencia un conocimiento amplio sobre los CDM, el recurso de prevención por excelencia para adolescentes en riesgo en el escenario local. Aborda las características que definen estos recursos como eje del trabajo socioeducativo en 
coordinación con otras áreas de prevención-protección como la educación formal o los itinerarios de acceso al empleo. Así mismo, aporta una serie de claves para el trabajo con estos adolescentes en el marco comunitario.

\section{Agradecimientos}

La realización de la presente investigación se ha realizado gracias a la participación voluntaria y desinteresada de los y las diferentes profesionales que desarrollan sus competencias en los Centros de Día de Menores de la provincia de Valencia (España). Agradecemos también los comentarios y consideraciones de los evaluadores de este artículo porque ello contribuye a la rigurosidad del mismo en su versión final.

\section{Referencias}

Abebe, T., \& Bessell, S. (2014). Advancing ethical research with children: Critical reflections on ethical guidelines. Children's Geographies, 12(1), 126-133. https://doi.org/ 10.1080/14733285.2013.856077

Arruabarrena, Mํ. I. (2001). Detección, valoración y toma de decisiones en las diferentes situaciones de desprotección infantil. Masson.

Beck, U. (1992). Risk society: Towards a new modernity. Sage.

Berger, P., \& Luckman, T. (1995). La construcción social de la realidad. Amorrortu.

Biosca, L., \& Casas, F. (Comps.) (1986). Jornades sobre els centres diürns per a la infancia $i$ adolescéncia. Diputació de Barcelona.

Buttram, J. (1990). Focus group: A starting point for needs assessment. Eric.

Castro, C., \& Rodríguez, E. (2016). Intervención social con adolescentes: necesidades y recursos. Trabajo Social Hoy, (77), 7-23. https://doi.org/10.12960/TSH.2016.0001

Ciocanel, O., Power, K., Eriksen, A., \& Gillings, K. (2017). Effectiveness of positive youth development interventions: A meta-analysis of randomized controlled trials. Journal of Youth and Adolescence, 46(3), 483-504. https://doi.org/10.1007/s10964-016-0555-6

Coulthard, M. (1998). Introduction to discourse analysis. Longman.

Denzin, N. K. (1970). Sociological methods. Aldine Publishing Company.

Ferrero, P. (2012). Los Centros de Día de Atención a la Infancia en la Comunidad Valenciana. Quaderns de Ciéncies Socials, (23), 33-59. 
Figueiredo, G. (2016). Los jóvenes en favelas de Rio de Janeiro, Brasil: de la vulnerabilidad social a las oportunidades para el desarrollo humano. Ciência Saúde Coletiva, 21(8), 2437-2450. https://doi.org/10.1590/1413-81232015218.01622015

Funes, J. (2009). Nueve ideas clave para educar en la adolescencia. Graó.

Generalitat Valenciana. (2018). Ley 26. De derechos y garantías de la infancia y la adolescencia. https://bit.ly/33NMoQi

González, F. (2006). Estudio de los perfiles de las familias en situación de riesgo social: programas de ayudas P.E.R. y P.E.P. del Ayuntamiento de Valencia [Tesis doctoral no publicada]. Universitat de Valencia.

Gresham, F. (2016). Social skills assessment and intervention for children and youth. Cambridge Journal of Education, 46(3), 319-332. https://doi.org/10.1080/0305764X.2016.1195788

Jiménez, A. (2016). Propuesta de un sistema de indicadores para la intervención socioeducativa y la gestión en los Centros de Día de Menores en la Comunidad Valenciana [Tesis doctoral no publicada]. Universitat de Valencia.

Kvale, S. (2011). Las entrevistas en investigación cualitativa. Morata.

Lederman, L. (1990). Assessing educational effectiveness: The focus group interview as a technique for data collection. Communication Education, 39(2), 117-127. https:// doi.org/10.1080/03634529009378794

Legreco, M., \& Tracey, S. (2009). Discourse tracing as qualitative practice. Qualitative Inquiry, 15(9), 1516-1543. https://doi.org/10.1177/1077800409343064

Martínez, L. (2002). Las instituciones de atención social al menor en la ciudad de Albacete. Instituto de Estudios Albacetenses Don Juan Manuel de la Excma. Diputación de Albacete.

Melendro, M., De Juanas, A., \& Rodríguez, A. (2017). Déficits en la intervención socioeducativa con familias de adolescentes en riesgo de exclusión. Bordón. Revista de Pedagogía, 69(1), 123-138. https://doi.org/10.13042/Bordon.2016.48596

Montoliu, A. (2013). Logros de los Centros de Día de la Comunidad Valenciana. Coordinadora de Menores de la Comunidad Valenciana.

Morse, J. (1995). The significance of saturation. Qualitative Health Research, 5(2), 147-149.

Navarro-Pérez, J. J. (2014). Estilos de socialización en adolescentes en conflicto con la ley con perfil de ajuste [Tesis doctoral no publicada]. Universitat de Valencia.

Navarro-Pérez, J. J., \& Pastor, E. (2018). De los riesgos en la socialización global: adolescentes en conflicto con la ley con perfil de ajuste social. Revista de Ciencias Sociales Convergencia, (76), 119-145. https://doi.org/10.29101/crcs.v25i76.4442 
Navarro-Pérez, J. J., \& Puig, M. (2010). El valor de la educación afectiva con niños en situación de vulnerabilidad acogidos en instituciones de protección: un modelo de trabajo social basado en la cotidianidad. Revista de Servicios Sociales y Política Social, (90), 65-84.

Nussbaum, M. (2012). Crear capacidades: propuesta para el desarrollo humano. Paidós.

Oberle, E., Schonert-Reichl, K., \& Zumbo, B. (2011). Life satisfaction in early adolescence: Personal, neighborhood, school, family, and peer influences. Journal of Youth and Adolescence, (40), 889-901. https://doi.org/10.1007/s10964-010-9599-1

Orrego, J. M., Paino, M., \& Fonseca, E. (2015). Programa educativo Trampolín para adolescentes con problemas graves del comportamiento: perfil de sus participantes y efecto de la intervención. Aula Abierta, 44(1), 38-45. https://doi.org/10.1016/j.aula.2015.06.001

Parker, A., \& Tritter, J. (2007). Focus group method and methodology: Current practice and recent debate. International Journal of Research \& Method in Education, 29(1), 23-37. https://doi.org/10.1080/01406720500537304

Perdomo, J., \& Varela, J. (2015). Metodología para la medición de riesgos sociales. Paradigmas, $7(1), 2-16$.

Ruiz, A. C., \& Palma, M. (2019). Prevención del abandono escolar temprano: aportaciones desde el trabajo social. Prospectiva. Revista de Trabajo Social e Intervención Social, (27), 139-158. https://doi.org/10.25100/prts.voi27.6569

Sánchez, J. (2011). Un recurso de integración social para niños/as, adolescentes y familias en situación de riesgo: los Centros de Día de Atención a Menores [Tesis doctoral no publicada]. Universidad de Granada.

Santibañez, R., \& Laespada, T. (2016). Necesidades socioeducativas de la infancia en Bilbao: una mirada transversal desde el territorio. Pedagogía Social. Revista Interuniversitaria, (28), 25-40. https://doi.org/10.7179/PSRI_2016.28.03

Steinberg, L., Icenogle, G., Shulman, E. P., Breiner, K., Chein, J., Bacchini, D., \& Takash, H. M. (2017). Around the world, adolescence is a time of heightened sensation seeking and immature self-regulation. Developmental Science, 21(2), 1-13. https://doi.org/10.1111/ desc. 12532

Tur, A. M., Llorca, A., Malonda, E., Samper, P., \& Mestre, M. V. (2016). Empatía en la adolescencia: relaciones con razonamiento moral prosocial, conducta prosocial y agresividad. Acción Psicológica, 13(2), 3-14. https://doi.org/10.5944/ap.13.2.17802

Uceda, X. (2011). Adolescentes en conflicto con la ley. Una aproximación comunitaria: trayectorias, escenarios e itinerarios [Tesis doctoral no publicada]. Universitat de Valencia. 
Uceda, X., \& Navarro-Pérez, J. J. (2013). La política de reeducación en la Comunitat Valenciana: un análisis de las prácticas educativas de los Centros de Justicia Juvenil. Alternativas. Cuadernos de Trabajo Social, (20), 57-77.

Vallejo, M., Moreno, M. C., \& Ríos, M. L. (2017). Sentido de comunidad, fatalismo y participación en contextos de crisis socioeconómica. Psychosocial Intervention, 26(1), 1-7. https://doi.org/10.1016/j.psi.2016.10.002

Wengraf, T. (2012). Qualitative research interviewing. Sage.

Zambrano, A., Muñoz, J., \& Andrade, C. (2015). El desafío de incorporar las redes institucionales y comunitarias en la intervención con adolescentes infractores: una investigación acción en tres regiones del sur de Chile. Universitas Psychologica, 14(4). 1371-1386. https://doi.org/10.11144/Javeriana.up14-4.diri 